\title{
Purification of the recombinant enhanced green fluorescent protein from Escherichia coli using alcohol + salt aqueous two-phase systems
}

\begin{abstract}
The enhanced green fluorescent protein (EGFP) is widely used as a marker in life science. Currently, purifications of the EGFP mostly involve chromatographic methods, which are multistep, time-consuming and costly. In the present study, the recombinant EGFP expressed in Escherichia coli was purified using an economic aqueous two-phase system (ATPS). Shortchain aliphatic alcohol and organic salt were chosen as the phase-forming components owing to their recyclability and biodegradability, respectively. The partition behaviour of EGFP was evaluated under the varying conditions of ATPS, including types and concentrations of phaseforming components, feedstock concentration, and $\mathrm{pH}$. In an optimal primary 2-g ATPS comprising 1-propanol and tripotassium citrate, a high recovery of EGFP (i.e., 92.1\%) was attained in the salt-rich bottom phase. To facilitate the easy recovery of purified EGFP, a secondary ATPS was used to back extract the EGFP to a new top ethanol-rich top phase, and the ethanol was removed from the purified EGFP via evaporation. The 1-propanol and ethanol used in the primary and secondary ATPSs (i.e. 20-g system) were successfully recycled in three successive rounds of EGFP purification, yielding the average EGFP purification factor of 11.34 and $75.7 \%$ of EGFP yield. The purification of EGFP using the two stages of alcohol + salt ATPSs is efficient in terms of operation time, cost and process scalability.
\end{abstract}

Keyword: Enhanced green fluorescent protein; Aqueous two-phase systems; Alcohol; Salt; Back extraction 